\title{
PRECISE SPECTRAL ASYMPTOTICS FOR NONAUTONOMOUS LOGISTIC EQUATIONS OF POPULATION DYNAMICS IN A BALL
}

\author{
TETSUTARO SHIBATA
}

Received 17 August 2004

We consider the semilinear elliptic eigenvalue problem $-\Delta u+k(|x|) u^{p}=\lambda u, u>0$ in $B_{R}$, $u=0$ on $\partial B_{R}$, where $p>1$ is a constant, $B_{R}:=\left\{x \in \mathbf{R}^{N}:|x|<R\right\}(N \geq 1)$, and $\lambda>0$ is a parameter. We investigate the global structure of the branch of $\left(\lambda, u_{\lambda}\right)$ of bifurcation diagram from a point of view of $L^{2}$-theory. To do this, we establish a precise asymptotic formula for $\lambda=\lambda(\alpha)$ as $\alpha \rightarrow \infty$, where $\alpha:=\left\|u_{\lambda}\right\|_{2}$.

\section{Introduction}

We consider the semilinear nonautonomous logistic equation of population dynamics

$$
\begin{gathered}
-\Delta u+k(|x|) u^{p}=\lambda u \quad \text { in } B_{R}, \\
u>0 \quad \text { in } B_{R}, \\
u=0 \quad \text { on } \partial B_{R},
\end{gathered}
$$

where $p>1$ is a constant, $B_{R}:=\left\{x \in \mathbf{R}^{N}:|x|<R\right\}(N \geq 1)$ and $\lambda>0$ is a parameter.

We assume the following conditions.

(A.1) $k(r) \in C^{2}([0, R])$ and $k(r)>0$ for $r \in[0, R]$.

(A.2) $d k(r) / d r>0$ for $r \in[0, R]$.

(A.3) Let $K(r):=k(r)^{-1 /(p-1)}$. Then $-\Delta K(|x|) \geq 0$ for $x \in B_{R}$.

The typical example is $k(r)=(1+r)^{2}, p=3$ and $N \geq 3$.

The equation (1.1)-(1.3) is a model of population density for some species when $p=$ 2. Here, $\lambda>0$ is regarded as the reciprocal number of its diffusion rate, and $k$ the effect of crowding for the species. Since (1.1)-(1.3) is motivated by this biological background, it has been investigated by many authors. In particular, it is shown by the arguments in $[1,6]$ (we also refer to $[7,10,11,12]$ ) that:

(a) for each $\lambda>\lambda_{1}$, there exists a unique solution $\left(\lambda, u_{\lambda}\right) \in \mathbf{R}_{+} \times C^{2}\left(\bar{B}_{R}\right)$ of (1.1)-(1.3), where $\lambda_{1}$ is the first eigenvalue of $-\Delta$ with Dirichlet zero boundary condition,

(b) the set $\left\{\left(\lambda, u_{\lambda}\right), \lambda>\lambda_{1}\right\}$ gives all the solutions of (1.1)-(1.3) and is an unbounded curve of class $C^{1}$ in $\mathbf{R}_{+} \times C^{2}\left(\bar{B}_{R}\right)$ emanating from $\left(\lambda_{1}, 0\right)$. 
Therefore, one of the main interests to study (1.1)-(1.3) is to understand well this bifurcation diagram.

The purpose here is to study precisely the global structure of this bifurcation diagram from a view point of $L^{2}$-theory to know the relationship between the reciprocal parameter $\lambda$ of the diffusion rate and the number of the population of the species. To do this, we parametrize the solution set by its $L^{2}$-norm as follows. For a given $\alpha>0$, we denote by $\left(\lambda(\alpha), u_{\alpha}\right) \in\left\{\lambda>\lambda_{1}\right\} \times C^{2}\left(\bar{B}_{R}\right)$ the solution pair of (1.1)-(1.3) with $\left\|u_{\alpha}\right\|_{L^{2}\left(B_{R}\right)}=\alpha$, which uniquely exists (i.e. $\left.u_{\alpha}=u_{\lambda(\alpha)}\right)$. We call the graph $\lambda=\lambda(\alpha)(\alpha>0)$ the $L^{2}$-bifurcation diagram of (1.1)-(1.3).

To gain a clearer picture of the $L^{2}$-bifurcation diagram locally and globally, several attempts have been made to study the asymptotic behavior of $\lambda(\alpha)$ as $\alpha \rightarrow 0$ and $\alpha \rightarrow \infty$. For the works in this direction, we refer to $[2,3,4,5,9,14,15,16,17]$ and the references therein. In particular, $[3,4]$ obtained the asymptotic formulas for $\lambda(\alpha)$ as $\alpha \rightarrow 0$ by using a variational method. On the other hand, if we focus our attention to the behavior of $\lambda(\alpha)$ as $\alpha \rightarrow \infty$, then since

$$
u_{\lambda}(x) / \lambda^{1 /(p-1)} \longrightarrow K(|x|)
$$

uniformly on any compact subset in $B_{R}$ as $\lambda \rightarrow \infty$ (cf. Lemma 2.1 in Section 2), we easily see from (1.4) that

$$
\alpha^{2}=\left\|u_{\alpha}\right\|_{2}^{2}=(1+o(1)) \lambda(\alpha)^{2 /(p-1)} \int_{B_{R}} K(|x|)^{2} d x .
$$

This implies

$$
\lambda(\alpha)=A(K) \alpha^{p-1}+o\left(\alpha^{p-1}\right)
$$

where $A(K)=\|K\|_{L^{2}\left(B_{R}\right)}^{1-p}$. It is therefore natural to ask: what is the exact second term of $\lambda(\alpha)$ as $\alpha \rightarrow \infty$ ?

Before answering this question, we recall some known results for the autonomous case. Let

$$
\begin{gathered}
C_{0}:=\int_{0}^{1} \sqrt{\frac{p-1}{p+1}-s^{2}+\frac{2}{p+1} s^{p+1}} d s, \\
B(K):=\frac{p+3}{2} C_{0} A(K)^{(p+3) /(2(p-1))} R^{N-1}\left|S^{N-1}\right|,
\end{gathered}
$$

where $\left|S^{N-1}\right|$ is the measure of the unit sphere in $\mathbf{R}^{N}$.

Theorem $1.1[15,16]$. (i) Let $N=1$ and $k \equiv 1$. Furthermore, replace $B_{R}$ by $(0,1)$ in $(1.1)$ (1.3). Then as $\alpha \rightarrow \infty$

$$
\lambda(\alpha)=\alpha^{p-1}+(p+3) C_{0} \alpha^{(p-1) / 2}+O(1) .
$$

(ii) Let $k \equiv 1$ in (1.1)-(1.3). Then as $\alpha \rightarrow \infty$

$$
\lambda(\alpha)=A(1) \alpha^{p-1}+B(1) \alpha^{(p-1) / 2}+o\left(\alpha^{(p-1) / 2}\right) .
$$


We remark that $m$-th $(m \in \mathbf{N})$ term of $\lambda(\alpha)$ has been obtained in [15] for the case $N=1$. Furthermore, if $k \equiv 1$, then (1.9) has been extended to the case of general bounded domain (cf. [17]).

On the other hand, for the nonautonomous case, only the following result seems to have been given.

Theorem 1.2 [14, Theorem 1]. Let $N=1$ and $R=1$ in (1.1)-(1.3). Then the following asymptotic formula holds as $\alpha \rightarrow \infty$ :

$$
\lambda(\alpha)=A(K) \alpha^{p-1}+O\left(\alpha^{(p-1) / 2}\right) .
$$

We see that for the nonautonomous case, not the exact second term has been obtained even if $N=1$.

The main aim here, therefore, is to establish the exact second term for the nonautonomous case when $N \geq 1$.

Now we state our result.

Theorem 1.3. Let $C(K):=K(R)^{2} B(K)$. Then as $\alpha \rightarrow \infty$

$$
\lambda(\alpha)=A(K) \alpha^{p-1}+C(K) \alpha^{(p-1) / 2}+o\left(\alpha^{(p-1) / 2}\right) .
$$

Comparing (1.11) with (1.9), we find that the leading term of $\lambda(\alpha)$ is affected by the value of $K$ in $B_{R}$, while the second term is affected also by $K(R)$, the value of $K$ at the boundary.

We explain briefly the reason why the nonautonomous equation is difficult to treat. When we consider the autonomous case, to obtain the generalization of (1.9) in [17] for general bounded domain $\Omega$, the Pohozaev identity and the precise study of the asymptotic behavior of $u_{\lambda}$ near $\partial \Omega$ have played crucial roles. Unfortunately, the Pohozaev identity is not useful any more for nonautonomous case. Furthermore, the precise analysis of the asymptotic behavior of $u_{\lambda}$ near $\partial \Omega$ is quite difficult to study when the equation is nonautonomous and $\Omega$ is a general bounded domain. Therefore, we restrict our attention to the case where $\Omega=B_{R}$ here as a first step to investigate the nonautonomous cases. The foreseeable extension of this research would certainly be to investigate the case where $\Omega$ is a general bounded domain.

The approach of the proof of Theorem 1.3 is based on the arguments developed in [16], since in this case, by (A.2), we see from [8] that (1.1)-(1.3) is equivalent to the ODE

$$
\begin{gathered}
u^{\prime \prime}(r)+\frac{N-1}{r} u^{\prime}(r)+\lambda u(r)-k(r) u(r)^{p}=0, \quad 0<r<R, \\
u(r)>0, \quad 0 \leq r<R, \\
u^{\prime}(0)=u(R)=0 .
\end{gathered}
$$

In [16], the key ingredient for obtaining the second term of $\lambda(\alpha)$ in (1.9) is to find the asymptotic formulas for $\left\|\nabla u_{\alpha}\right\|_{2},\left\|u_{\alpha}\right\|_{p+1}^{p+1}$ and $\int_{0}^{R}(N-1) u_{\alpha}^{\prime}(s)^{2} / s d s$ as $\alpha \rightarrow \infty$. However, if $k \neq 1$, then clearly these terms are affected by $k$ and we encounter several difficulties caused by $k$. Therefore, the calculation of these terms are more complicated than that to 
obtain (1.9) and in particular, the effect from the boundary on these terms must be taken into careful consideration.

\section{Proof of Theorem 1.3}

For convenience, we identify (1.1)-(1.3) with (1.12)-(1.14). Furthermore, for the notation of the solution pair of (1.1)-(1.3) and (1.12)-(1.14), we use both $\left(\lambda, u_{\lambda}\right)$ and $\left(\lambda(\alpha), u_{\alpha}\right)$. Furthermore, we write $A=A(K)$ for simplicity.

Lemma 2.1. Let an arbitrary $0<R_{0}<R$ be fixed. Then as $\lambda \rightarrow \infty$

$$
\frac{u_{\lambda}(r)}{\lambda^{1 /(p-1)}}=K(r)(1+o(1))
$$

uniformly on $\left[0, R_{0}\right]$.

Proof. Let an arbitrary $x_{0} \in \bar{B}_{R_{0}}$ be fixed. Furthermore, let $r_{0}=\left|x_{0}\right|$ and $B_{x_{0}, \delta}:=\{\mid x-$ $\left.x_{0} \mid<\delta\right\} \subset B_{R}$. By (A.2), we see that for $x \in B_{x_{0}, \delta}$

$$
k\left(r_{0}-\delta\right)<k(|x|)<k\left(r_{0}+\delta\right)
$$

Let $w_{\lambda}$ be a unique solution of

$$
\begin{gathered}
-\Delta w+k(|x|) w^{p}=\lambda w \quad \text { in } B_{x_{0}, \delta} \\
w>0 \quad \text { in } B_{x_{0}, \delta} \\
w=0 \quad \text { on } \partial B_{x_{0}, \delta} .
\end{gathered}
$$

Then a comparison and maximum principle show that $u_{\lambda} \geq w_{\lambda}$ in $B_{x_{0}, \delta}$. Furthermore, let $v_{\lambda}$ be a unique solution of

$$
\begin{gathered}
-\Delta v+k\left(r_{0}+\delta\right) v^{p}=\lambda v \quad \text { in } B_{x_{0}, \delta} \\
v>0 \quad \text { in } B_{x_{0}, \delta} \\
v=0 \quad \text { on } \partial B_{x_{0}, \delta} .
\end{gathered}
$$

Then by (2.2), for $x \in B_{x_{0}, \delta}$, we obtain

$$
-\Delta v_{\lambda}+k(|x|) v_{\lambda}^{p}-\lambda v_{\lambda}=\left(k(|x|)-k\left(r_{0}+\delta\right)\right) v_{\lambda} \leq 0
$$

This implies that $v_{\lambda}$ is a subsolution of (2.3). Furthermore, since a constant function $\left(\lambda / k\left(r_{0}+\delta\right)\right)^{1 /(p-1)}$ is a supersolution of (2.4), by (2.2), we see that for $x \in B_{x_{0}, \delta}$

$$
v_{\lambda}(x) \leq\left(\frac{\lambda}{k\left(r_{0}+\delta\right)}\right)^{1 /(p-1)} \leq W_{\lambda}(x):=\left(\frac{\lambda}{k(|x|)}\right)^{1 /(p-1)}
$$


By (A.3), we see that $W_{\lambda}$ is a super-solution of both (1.1)-(1.3) and (2.3). Therefore, by $(2.6)$

$$
\begin{gathered}
v_{\lambda}(x) \leq w_{\lambda}(x) \leq W_{\lambda}(x), \quad x \in B_{r_{0}, \delta} \\
u_{\lambda}(x) \leq W_{\lambda}(x), \quad x \in B_{R} .
\end{gathered}
$$

Since $u_{\lambda} \geq w_{\lambda}$, consequently,

$$
v_{\lambda}(x) \leq w_{\lambda}(x) \leq u_{\lambda}(x) \leq W_{\lambda}(x), \quad x \in B_{x_{0}, \delta}
$$

On the other hand, it is known (cf. [6]) that for $x \in \bar{B}_{x_{0}, \delta / 2}=\left\{\left|x-x_{0}\right| \leq \delta / 2\right\}$, as $\lambda \rightarrow \infty$

$$
\frac{v_{\lambda}(x)}{\lambda^{1 /(p-1)}} \longrightarrow k\left(r_{0}+\delta\right)^{-1 /(p-1)}
$$

uniformly. Since $\bar{B}_{R_{0}}$ is covered by finite number of balls with radius $\delta / 2$ such as $B_{x_{0}, \delta / 2}$, and $\delta$ is arbitrary, by (2.8) and (2.9), we obtain our conclusion.

Corollary 2.2. As $\lambda \rightarrow \infty$

$$
\left\|u_{\lambda}\right\|_{\infty}=\lambda^{1 /(p-1)}(K(0)+o(1)) .
$$

Lemma 2.3. As $\lambda \rightarrow \infty$

$$
\int_{0}^{R} \frac{N-1}{r} u_{\lambda}^{\prime}(r)^{2} d r=o\left(\lambda^{(p+1) /(p-1)}\right) .
$$

Proof. Multiply (1.12) by $u_{\lambda}^{\prime}(r)$. Then

$$
\left\{u_{\lambda}^{\prime \prime}(r)+\frac{N-1}{r} u_{\lambda}^{\prime}(r)+\lambda u_{\lambda}(r)-k(r) u_{\lambda}(r)^{p}\right\} u_{\lambda}^{\prime}(r)=0 .
$$

This implies

$$
\begin{aligned}
\frac{1}{2} u_{\lambda}^{\prime}(r)^{2} & +\int_{0}^{r} \frac{N-1}{s} u_{\lambda}^{\prime}(s)^{2} d s+\frac{1}{2} \lambda u_{\lambda}(r)^{2} \\
& -\frac{1}{p+1} k(r) u_{\lambda}(r)^{p+1}+\int_{0}^{r} \frac{1}{p+1} k^{\prime}(s) u_{\lambda}(s)^{p+1} d s \\
= & \frac{1}{2} u_{\lambda}^{\prime}(R)^{2}+\int_{0}^{R} \frac{N-1}{r} u_{\lambda}^{\prime}(r)^{2} d s+\int_{0}^{R} \frac{1}{p+1} k^{\prime}(r) u_{\lambda}(r)^{p+1} d r \\
= & \frac{1}{2} \lambda\left\|u_{\lambda}\right\|_{\infty}^{2}-\frac{1}{p+1} k(0)\left\|u_{\lambda}\right\|_{\infty}^{p+1} .
\end{aligned}
$$

We fix $0<R_{0}<R$. Then by Lemma 2.1, for $0 \leq r \leq R_{0}$

$$
\begin{aligned}
\int_{0}^{r} k^{\prime}(s) u_{\lambda}(s)^{p+1} d s & =(1+o(1)) \lambda^{(p+1) /(p-1)} \int_{0}^{r} k^{\prime}(s) K(s)^{p+1} d s \\
& =(1+o(1)) \lambda^{(p+1) /(p-1)} \int_{k(0)}^{k(r)} \theta^{-(p+1) /(p-1)} d \theta \\
& =-\frac{p-1}{2}(1+o(1)) \lambda^{(p+1) /(p-1)}\left(K(r)^{2}-K(0)^{2}\right) .
\end{aligned}
$$


By this, Lemma 2.1 and Corollary 2.2, for $0 \leq r \leq R_{0}$, we obtain

$$
\begin{aligned}
L_{\lambda}(r):= & \frac{1}{2} \lambda\left\|u_{\lambda}\right\|_{\infty}^{2}-\frac{1}{p+1} k(0)\left\|u_{\lambda}\right\|_{\infty}^{p+1}-\frac{1}{2} \lambda u_{\lambda}(r)^{2} \\
& +\frac{1}{p+1} k(r) u_{\lambda}(r)^{p+1}-\frac{1}{p+1} \int_{0}^{r} k^{\prime}(s) u_{\lambda}(s)^{p+1} d s \\
= & \frac{1}{2}(1+o(1)) \lambda^{(p+1) /(p-1)} K(0)^{2}-\frac{1}{p+1}(1+o(1)) K(0)^{2} \lambda^{(p+1) /(p-1)} \\
& -\frac{1}{2}(1+o(1)) \lambda^{(p+1) /(p-1)} K(r)^{2}+\frac{1}{p+1}(1+o(1)) \lambda^{(p+1) /(p-1)} K(r)^{2} \\
& +\frac{p-1}{2(p+1)}(1+o(1)) \lambda^{(p+1) /(p-1)}\left(K(r)^{2}-K(0)^{2}\right) \\
= & o\left(\lambda^{(p+1) /(p-1)}\right) .
\end{aligned}
$$

By this and (2.13), we obtain

$$
\int_{0}^{R_{0}} \frac{N-1}{s} u_{\lambda}^{\prime}(s)^{2} d s \leq L_{\lambda}(r)=o\left(\lambda^{(p+1) /(p-1)}\right) .
$$

Multiply $(1.1)$ by $u_{\lambda}$. Then integration by parts along with Lemma 2.1 implies that as $\lambda \rightarrow \infty$

$$
\left\|\nabla u_{\lambda}\right\|_{2}^{2}=\lambda\left\|u_{\lambda}\right\|_{2}^{2}-\int_{B_{R}} k(|x|) u_{\lambda}(x)^{p+1} d x=o\left(\lambda^{(p+1) /(p-1)}\right) .
$$

By this, for $\lambda \gg 1$, we obtain

$$
\begin{aligned}
\int_{R_{0}}^{R} \frac{N-1}{s} u_{\lambda}^{\prime}(s)^{2} d s & \leq \frac{N-1}{R_{0}^{N}} \int_{R_{0}}^{R} s^{N-1} u_{\lambda}^{\prime}(s)^{2} d s \\
& \leq C\left\|u_{\lambda}\right\|_{2}^{2}=o\left(\lambda^{(p+1) /(p-1)}\right) .
\end{aligned}
$$

By this and (2.16), we obtain our conclusion. Thus the proof is complete.

Lemma 2.4. As $\lambda \rightarrow \infty$

$$
\left\|\nabla u_{\lambda}\right\|_{2}^{2}=K(R)^{2} R^{N-1}\left|S^{N-1}\right| C_{0} \lambda^{(p+3) /(2(p-1))}(1+o(1)) .
$$


Proof. Let an arbitrary $0<\delta \ll 1$ be fixed. We first show that for $\lambda \gg 1$

$$
\int_{0}^{R-\delta} r^{N-1} u_{\lambda}^{\prime}(r)^{2} d r=o\left(\lambda^{(p+3) /(2(p-1))}\right)
$$

By (2.13) and (2.15), for $r \in[0, R-\delta]$,

$$
\frac{1}{2} u_{\lambda}^{\prime}(r)^{2} \leq L_{\lambda}(r)
$$

By this, (2.15), Lemma 2.1 and Corollary 2.2, we obtain

$$
\begin{aligned}
\int_{0}^{R-\delta} r^{N-1} u_{\lambda}^{\prime}(r)^{2} d r & \leq R^{N-1} \int_{0}^{R-\delta} u_{\lambda}^{\prime}(r)^{2} d r \\
& \leq R^{N-1} \int_{0}^{R-\delta}-\sqrt{L_{\lambda}(r)} u_{\lambda}^{\prime}(r) d r \\
& =o\left(\lambda^{(p+1) /(2(p-1))}\right) \int_{0}^{R-\delta}-u_{\lambda}^{\prime}(r) d r \\
& =o\left(\lambda^{(p+1) /(2(p-1))}\right)\left(\left\|u_{\lambda}\right\|_{\infty}-u_{\lambda}(R-\delta)\right) \\
& =o\left(\lambda^{(p+3) /(2(p-1))}\right) .
\end{aligned}
$$

Next, by Lemma 2.3, (2.13) and (2.14), for a fixed $0 \leq r<R$, as $\lambda \rightarrow \infty$

$$
\begin{aligned}
\frac{1}{2} u_{\lambda}^{\prime}(r)^{2}= & \frac{1}{2} \lambda\left\|u_{\lambda}\right\|_{\infty}^{2}-\frac{1}{p+1} k(0)\left\|u_{\lambda}\right\|_{\infty}^{p+1}-\frac{1}{2} \lambda u_{\lambda}(r)^{2} \\
& +\frac{1}{p+1} k(r) u_{\lambda}(r)^{p+1}+\frac{p-1}{2(p+1)} \lambda^{(p+1) /(p-1)}\left(K(r)^{2}-K(0)^{2}\right) \\
& +o\left(\lambda^{(p+1) /(p-1)}\right) .
\end{aligned}
$$

By this, (A.2) and Lemma 2.1, for a fixed $r \in[R-\delta, R)$

$$
\begin{aligned}
\frac{1}{2} u_{\lambda}^{\prime}(r)^{2} \leq & \frac{1}{2} M_{\lambda}(r) \\
:= & \frac{p-1}{2(p+1)} K(R-\delta)^{2} \lambda^{(p+1) /(p-1)}-\frac{1}{2} \lambda u_{\lambda}(r)^{2} \\
& +\frac{1}{p+1} k(R) u_{\lambda}(r)^{p+1}+o\left(\lambda^{(p+1) /(p-1)}\right) .
\end{aligned}
$$


By this, we obtain

$$
\begin{aligned}
\limsup _{\lambda \rightarrow \infty} & \lambda^{-(p+3) /(2(p-1))} \int_{R-\delta}^{R} r^{N-1} u_{\lambda}^{\prime}(r)^{2} d r \\
\leq & \limsup _{\lambda \rightarrow \infty} \lambda^{-(p+3) /(2(p-1))} R^{N-1} \int_{R-\delta}^{R}-\sqrt{M_{\lambda}(r)} u_{\lambda}^{\prime}(r) d r \\
= & \limsup _{\lambda \rightarrow \infty} \lambda^{-(p+3) /(2(p-1))} R^{N-1} \\
& \times \int_{0}^{u_{\lambda}(R-\delta)} \sqrt{\frac{p-1}{p+1} K(R-\delta)^{2} \lambda(p+1) /(p-1)(1+o(1))-\lambda \theta^{2}+\frac{2}{p+1} k(R) \theta^{p+1}} d \theta \\
= & \limsup _{\lambda \rightarrow \infty} R^{N-1}\left(1+\epsilon_{1}(\delta)\right) \\
& \times \int_{0}^{u_{\lambda}(R-\delta) / \lambda^{1 /(p-1)}} \sqrt{\frac{p-1}{p+1} K(R)^{2}-s^{2}+\frac{2}{p+1} k(R) s^{p+1}} d s \\
\leq & R^{N-1}\left(1+\epsilon_{2}(\delta)\right) \\
& \times \int_{0}^{K(R-\delta)} \sqrt{\frac{p-1}{p+1} K(R)^{2}-s^{2}+\frac{2}{p+1} k(R) s^{p+1}} d s \quad(p u t s=K(R) t) \\
= & R^{N-1}\left(1+\epsilon_{3}(\delta)\right) K(R)^{2} \int_{0}^{1} \sqrt{\frac{p-1}{p+1}-t^{2}+\frac{2}{p+1} t t^{p+1}} d t,
\end{aligned}
$$

where $\epsilon_{1}(\delta), \epsilon_{2}(\delta), \epsilon_{3}(\delta) \rightarrow 0$ as $\delta \rightarrow 0$. By the same arguments as those above, we obtain

$$
\begin{aligned}
& \liminf _{\lambda \rightarrow \infty} \lambda^{-(p+3) /(2(p-1))} \int_{R-\delta}^{R} r^{N-1} u_{\lambda}^{\prime}(r)^{2} d r \\
& \quad \geq\left(1-\epsilon_{4}(\delta)\right) K(R)^{2}(R-\delta)^{N-1} \int_{0}^{1} \sqrt{\frac{p-1}{p+1}-s^{2}+\frac{2}{p+1} s^{p+1}} d s,
\end{aligned}
$$

where $\epsilon_{4}(\delta) \rightarrow 0$ as $\delta \rightarrow 0$. Since $\delta$ is arbitrary, by this and (2.25), we obtain

$$
\lim _{\lambda \rightarrow \infty} \lambda^{-(p+3) /(2(p-1))} \int_{R-\delta}^{R} r^{N-1} u_{\lambda}^{\prime}(r)^{2} d r=K(R)^{2} C_{0} R^{N-1} .
$$

By this and (2.22), we obtain (2.19). Thus the proof is complete. 
The following Corollary follows directly from (1.6) and Lemma 2.4.

Corollary 2.5. Let $C_{3}=K(R)^{2} R^{N-1}\left|S^{N-1}\right| A^{(p+3) /(2(p-1))} C_{0}$. Then as $\alpha \rightarrow \infty$

$$
\left\|\nabla u_{\alpha}\right\|_{2}^{2}=C_{3} \alpha^{(p+3) / 2}(1+o(1)) .
$$

We define the critical level $\eta(\alpha)$ by

$$
\eta(\alpha)=\left\|\nabla u_{\alpha}\right\|_{2}^{2}+\frac{2}{p+1} \int_{B_{R}} k(|x|) u_{\alpha}(x)^{p+1} d x .
$$

The following lemma is a variant of [16, Lemma 2.1].

Lemma 2.6. $d \eta(\alpha) / d \alpha=2 \lambda(\alpha) \alpha$.

Proof. We have

$$
\begin{aligned}
\frac{d \eta(\alpha)}{d \alpha} & =2 \int_{B_{R}} \nabla u_{\alpha} \cdot \nabla\left(\frac{d u_{\alpha}}{d \alpha}\right) d x+2 \int_{B_{R}} k(|x|) u_{\alpha}^{p} \frac{d u_{\alpha}}{d \alpha} d x \\
& =2 \int_{B_{R}}\left(-\Delta u_{\alpha}+k(|x|) u_{\alpha}^{p}\right) \frac{d u_{\alpha}}{d \alpha} d x \\
& =2 \lambda(\alpha) \int_{B_{R}} u_{\alpha} \frac{d u_{\alpha}}{d \alpha} d x=\lambda(\alpha) \frac{d\left\|u_{\alpha}\right\|_{2}^{2}}{d \alpha} \\
& =2 \lambda(\alpha) \alpha .
\end{aligned}
$$

Thus the proof is complete.

Proof of Theorem 1.3. By (2.17) and (2.29)

$$
\eta(\alpha)-\frac{2}{p+1} \lambda(\alpha) \alpha^{2}=\frac{p-1}{p+1}\left\|\nabla u_{\alpha}\right\|_{2}^{2} .
$$

We put

$$
\begin{aligned}
& \lambda_{1}(\alpha):=\lambda(\alpha)-A \alpha^{p-1} \\
& \eta_{1}(\alpha):=\eta(\alpha)-\frac{2}{p+1} A \alpha^{p+1} .
\end{aligned}
$$

Then it is easy to see from (1.6), Lemma 2.1 and Corollary 2.5 that as $\alpha \rightarrow \infty$

$$
\lambda_{1}(\alpha)=o\left(\alpha^{p-1}\right), \quad \eta_{1}(\alpha)=o\left(\alpha^{p+1}\right) .
$$

Furthermore, by (2.31), (2.32), (2.33), Corollary 2.5 and Lemma 2.6

$$
\begin{gathered}
\eta_{1}(\alpha)-\frac{2}{p+1} \lambda_{1}(\alpha) \alpha^{2}=\frac{p-1}{p+1}\left\|\nabla u_{\alpha}\right\|_{2}^{2}=\frac{p-1}{p+1} C_{3} \alpha^{(p+3) / 2}(1+o(1)), \\
\frac{d \eta_{1}(\alpha)}{d \alpha}=2 \lambda_{1}(\alpha) \alpha .
\end{gathered}
$$


By (2.35) and (2.36), we obtain

$$
\eta_{1}(\alpha)-\frac{\alpha}{p+1} \eta_{1}^{\prime}(\alpha)=\frac{p-1}{p+1} C_{3} \alpha^{(p+3) / 2}(1+o(1)) .
$$

We put $g(\alpha):=\eta_{1}(\alpha) / \alpha^{p+1}$. Then by (2.37),

$$
g^{\prime}(\alpha)=-(p-1) C_{3}(1+o(1)) \alpha^{-(p+1) / 2} .
$$

Since $g(\alpha) \rightarrow 0$ as $\alpha \rightarrow \infty$, we obtain

$$
g(\alpha)=-\int_{\alpha}^{\infty} g^{\prime}(s) d s=2 C_{3}(1+o(1)) \alpha^{(1-p) / 2} .
$$

This implies

$$
\eta_{1}(\alpha)=2 C_{3}(1+o(1)) \alpha^{(p+3) / 2}
$$

By this and (2.35), we obtain

$$
\frac{2}{p+1} \lambda_{1}(\alpha) \alpha^{2}=\frac{p+3}{p+1} C_{3} \alpha^{(p+3) / 2}(1+o(1)) .
$$

Since $(p+3) C_{3} / 2=C(K)$, this along with (2.32) implies (1.11). Thus the proof is complete.

\section{References}

[1] H. Berestycki, Le nombre de solutions de certains problèmes semi-linéaires elliptiques, J. Funct. Anal. 40 (1981), no. 1, 1-29 (French).

[2] J. Chabrowski, On nonlinear eigenvalue problems, Forum Math. 4 (1992), no. 4, 359-375.

[3] R. Chiappinelli, On spectral asymptotics and bifurcation for elliptic operators with odd superlinear term, Nonlinear Anal. 13 (1989), no. 7, 871-878.

[4] , Remarks on bifurcation for elliptic operators with odd nonlinearity, Israel J. Math. 65 (1989), no. 3, 285-292.

[5] Constrained critical points and eigenvalue approximation for semilinear elliptic operators, Forum Math. 11 (1999), no. 4, 459-481.

[6] J. M. Fraile, J. López-Gómez, and J. C. Sabina de Lis, On the global structure of the set of positive solutions of some semilinear elliptic boundary value problems, J. Differential Equations 123 (1995), no. 1, 180-212.

[7] J. García-Melián and J. Sabina de Lis, Stationary profiles of degenerate problems when a parameter is large, Differential Integral Equations 13 (2000), no. 10-12, 1201-1232.

[8] B. Gidas, W. M. Ni, and L. Nirenberg, Symmetry and related properties via the maximum principle, Comm. Math. Phys. 68 (1979), no. 3, 209-243.

[9] H.-P. Heinz, Nodal properties and variational characterizations of solutions to nonlinear SturmLiouville problems, J. Differential Equations 62 (1986), no. 3, 299-333.

[10] M. Holzmann and H. Kielhöfer, Uniqueness of global positive solution branches of nonlinear elliptic problems, Math. Ann. 300 (1994), no. 2, 221-241.

[11] P. H. Rabinowitz, A note on a nonlinear eigenvalue problem for a class of differential equations, J. Differential Equations 9 (1971), 536-548. 
[12] , Some global results for nonlinear eigenvalue problems, J. Functional Analysis 7 (1971), 487-513.

[13] T. Shibata, Asymptotic behaviour of variational eigenvalues of the nonlinear elliptic eigenvalue problem in a ball, Nonlinearity 7 (1994), no. 6, 1645-1653.

[14] Asymptotic formulas of eigenvalues and eigenfunctions of non-autonomous semilinear Sturm-Liouville problems, Math. Z. 219 (1995), no. 4, 485-498.

[15] _ Precise spectral asymptotics for nonlinear Sturm-Liouville problems, J. Differential Equations 180 (2002), no. 2, 374-394.

[16] _ Asymptotic formulas for boundary layers and eigencurves for nonlinear elliptic eigenvalue problems, Comm. Partial Differential Equations 28 (2003), no. 3-4, 581-600.

[17] - Precise spectral asymptotics for logistic equations of population dynamics, Bull. London Math. Soc. 36 (2004), no. 1, 59-64.

Tetsutaro Shibata: Applied Mathematics Research Group, Graduate School of Engineering, Hiroshima University, Higashi-Hiroshima, Hiroshima 739-8527, Japan

E-mail address: shibata@amath.hiroshima-u.ac.jp 


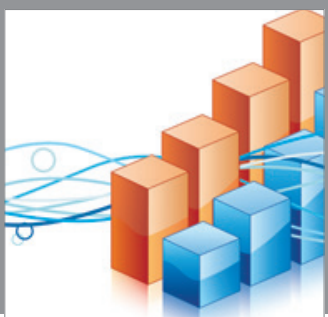

Advances in

Operations Research

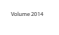

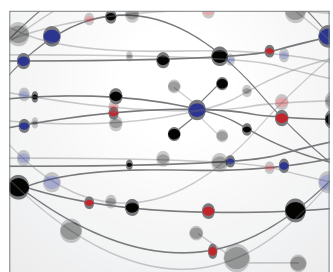

\section{The Scientific} World Journal
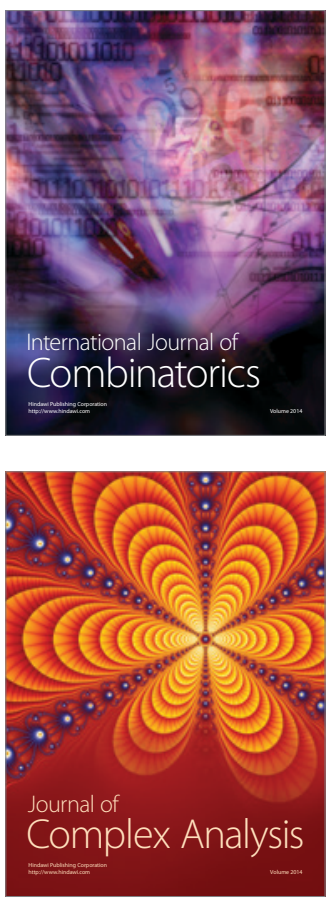

International Journal of

Mathematics and

Mathematical

Sciences
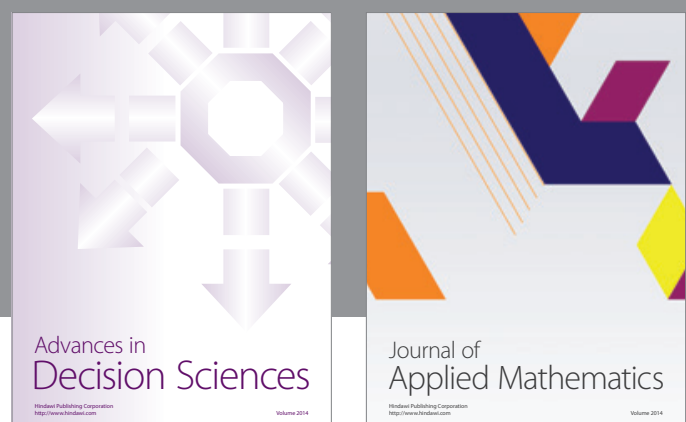

Journal of

Applied Mathematics
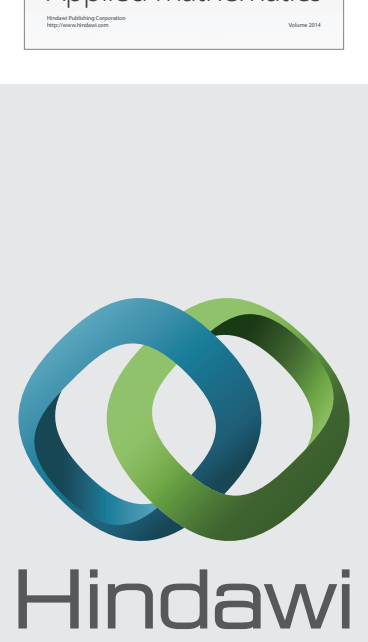

Submit your manuscripts at http://www.hindawi.com
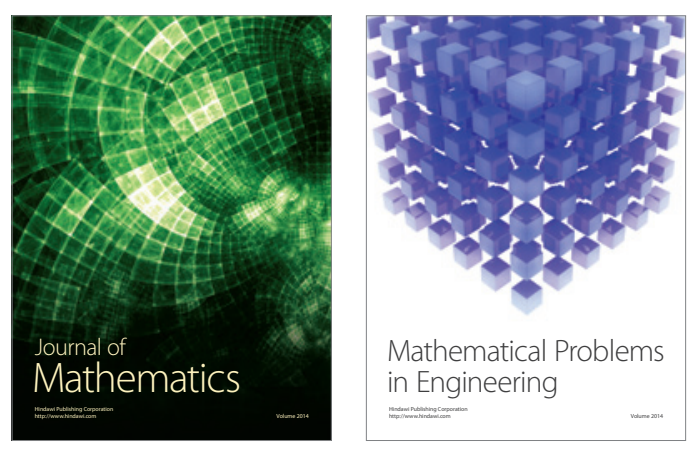

Mathematical Problems in Engineering
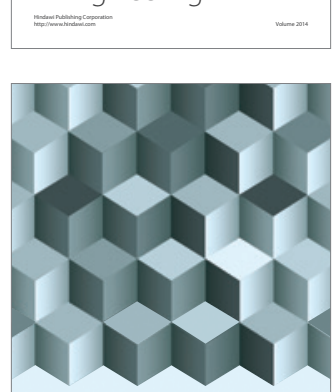

Journal of

Function Spaces
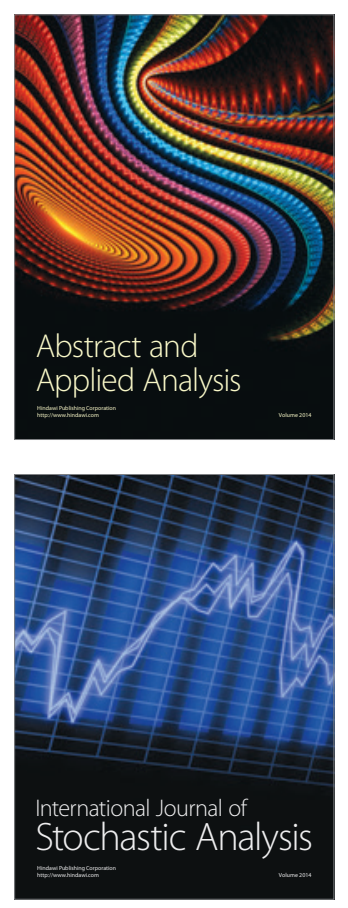

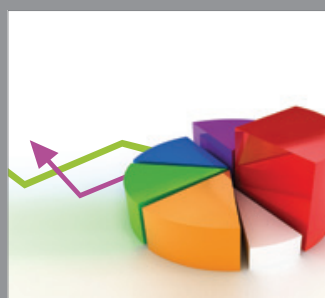

ournal of

Probability and Statistics

Promensencen
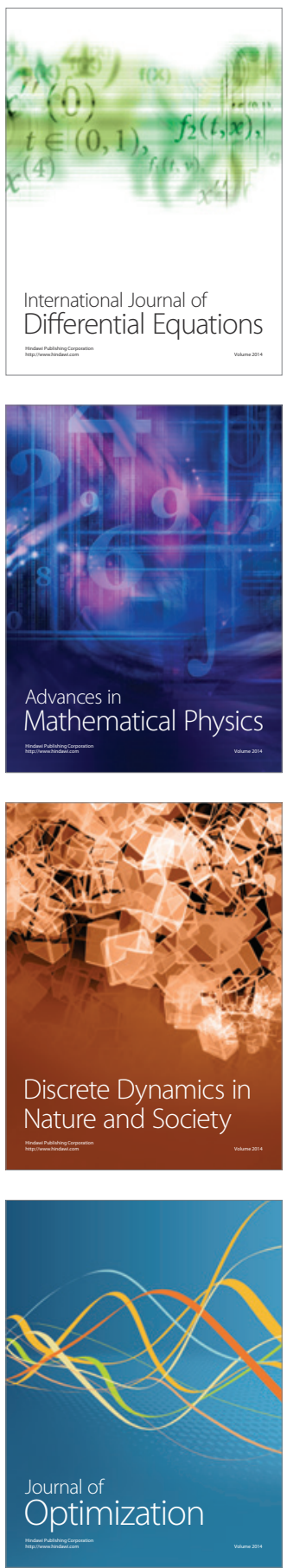